\title{
Satellite-Enabled Interactive Education: Scenarios and Systems Architectures
}

\author{
Tacha Serif $^{1}$, Lampros Stergioulas ${ }^{1}$, Gheorghita Ghinea ${ }^{1}$, Michalis Moatsos ${ }^{2}$, \\ Constantinos Makropoulos ${ }^{2}$, Sofia Tsekeridou ${ }^{3}$, and Thanassis Tiropanis ${ }^{3}$ \\ ${ }^{1}$ School of Information Systems, Computing and Mathematics \\ Brunel University, \\ Uxbridge, Middlesex, \\ UB8 3PH, UK \\ \{Tacha.Serif, Lampros.Stergioulas, \\ Gheorge. Ghinea @ @brunel .ac .uk \\ ${ }^{2}$ National Center of Scientific Research "Demokritos" \\ 15310 - Agia Paraskevi, Athens, Greece \\ \{mmoatsos, cmakr\} adat.demokritos.gr \\ ${ }^{3}$ Athens Information Technology (AIT) \\ P.O.Box 68 \\ $19.5 \mathrm{~km}$ Markopoulo Ave. \\ GR- 19002 Peania, Athens, Greece \\ \{sots, ttir\}ait.edu.gr
}

\begin{abstract}
There are specific sectors of the economy that can benefit from satellite-based tele-education. Areas, such as maritime and agriculture, share common needs for both broadband connectivity at remote geographical areas that cannot otherwise be covered, and for innovative content for tele-education purposes. Furthermore, each area has special requirements with regard to the type of content to be delivered. In this paper we propose a set of architectural designs and case scenarios that will realise such interactive end-to-end education systems based on satellite communications. Services requirements in this setting are also identified and discussed.
\end{abstract}

\section{Introduction}

This paper explores how innovative and efficient tele-education solutions can be offered over satellite to communities that would otherwise be excluded from the ongoing revolution in the area of tele-education and eLearning. That is not just because with satellite technology broadband accessibility will be offered to previously isolated communities, but because using innovative content and efficient tele-education platforms, such communities will be able to actively participate in an exchange of knowledge that is crucial for building a knowledge economy.

It is increasingly recognised that professional communities, which are distributed in remote places, will benefit significantly by using tele-education applications over satellite. In this study, we will consider the agricultural communities (farmers etc.) and the maritime communities (crew etc.) as two important exemplar groups for employing satellite based education systems. Farmers at remote areas, who often produce 
specialised products of designated origin, require continuous targeted training to increase or very often guarantee the quantity and quality of their production. Maritime workers also have continuous needs for tele-education and have been handicapped for many years by the lack of reliable and efficient network infrastructure that would help them reach this target. The structure of this paper is as follows: Section 2 presents an overview of distance learning systems; Section 3 identifies the user requirements of the two target communities; Section 4 describes three satellite network architectures that can be used for electronic learning purposes; Section 5 considers the main possible scenarios and their objectives. Section 6 details the services required for the implementation of satellite-based education systems. Finally, Section 7 draws the conclusions from this study.

\section{Networked Learning Environments for Distance Education}

Urdan and Weggen [1] define e-Learning as "the delivery of content via all electronic media, including the Internet, intranets, extranets, satellite broadcast, audio/video tape, interactive TV, and CD-ROM" In that way, e-Learning encompasses all currently available instruments, including digital or electronic tools, to facilitate learning and impart the contents of a course. Urdan and Weggen [1] also define e-Learning as a subset of distance learning, as depicted in the Fig. 1.

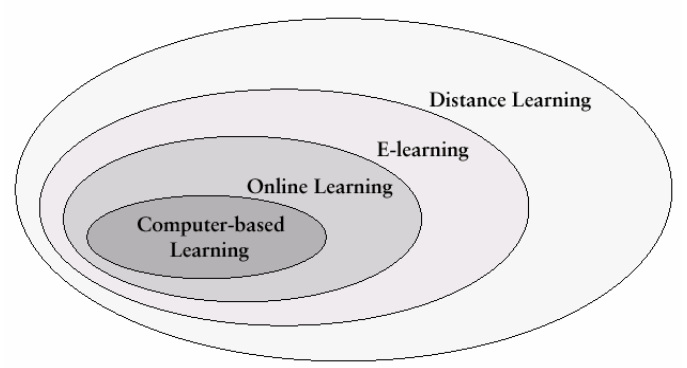

Fig. 1. Subsets of distance learning [1]

e-Learning is a term that nowadays covers almost any type of learning process based on information and communication technologies. E-learning is not just a course converted to be stored in a personal computer, but it rather represents a new mix of resources, interactivity and achievements; new structures for learning, a combination of teaching services using technological tools that adds value, anytime and anywhere [2]; [3]; [4].

As depicted in Fig. 2, e-learning is a composition of three main areas: Contents, Technologies and Services [5].

The Contents are the didactic units or reusable modules of the e-Learning process. The Technologies are the technological tools (platforms) that support the educational systems, including communication networks, protocols, and Learning Management Systems among others. The Services refer to the tracking and support offered to the students; e.g. Tutorials, periodic reports, assessments, course management, student motivation, course quality, etc. 


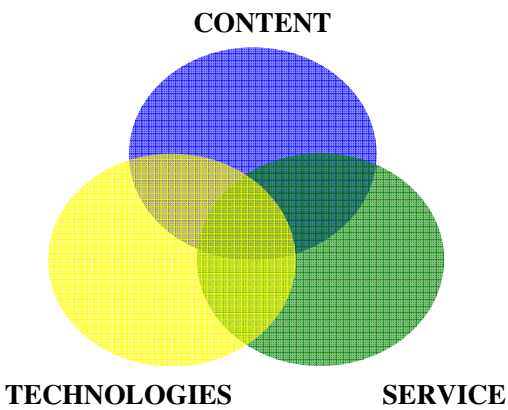

Fig. 2. E-Learning components [5]

e-Learning systems are defined as the platforms or systems offering features that support educative services through electronic media. These systems can be Learning Management Systems (LMS), Learning Content Management System (LCMS), Knowledge Pools, or Brokerage Systems.

A Learning Management System is an application running in a web server that is used to perform learning activities. It is also called a Platform or Virtual Campus. It is a place where students, tutors, teachers and coordinators connect through the Internet to download contents, examine the topics of each course, send e-mail to the teachers, chat with students, discuss in a forum, assist to a tutorial, etc.

A Learning Content Management System is a system that is independent or integrated into a LMS. It manages the learning contents. Once the contents are into the system, they can be assigned to each course, combined, downloaded, etc.

The Knowledge Pools are distributed repositories of metadata compatible with the Learning Object Metadata (LOM) standard which assure an efficient publication, search and restore of educative contents. An interesting characteristic of these servers is that they can be federated.

The Brokerage Systems store the description of the educative resources available locally or at remote locations.

\section{3 e-Learning System Requirements of User Groups}

This study focuses on the educational needs of agrarian and maritime communities that are in general remote communities with considerable networking and bandwidth constraints. It is often the case that access to e-learning services is not possible to remote villages or on a ship, since either no network is available or any existing bandwidth which might be available to these communities does not allow for fullyfeatured and rich multimedia-enabled services offering. Nevertheless, education and information updating is of vital importance to such communities to preserve their competitiveness and extend their activities or ease up their workload. Thus, a number of e-learning service requirements stem from the needs of such communities. Both require easy access to services, utilising common means and routines in the best possible way (natural interaction with new technology). However, with respect to educational content, the requirements differ according to the targeted community and the 
nature of their profession. Below we briefly summarize such requirements for each type of targeted communities.

The needs regarding educational content and thus services supporting such content are summarized as follows;

- Application of good agricultural practice codes.

- Cross compliance policies

- Quality products and products of geographical origin.

- New products

- Product processing, promotion and marketing

- Management issues

- Young farmers settling/installation assistance

- Special policies on rural women activities

- EU Policy changes in agriculture and the agrofood industry

Some of the above subjects constitute horizontal issues that concern all users. Others constitute vertical matters that concern a specific audience. In all cases, content preparation will have to be adjusted to the local needs and the products or sectors each area specializes in.

The content of a satellite-based tele-education service is designated primarily by the needs of the maritime vessel crew who aim to maintain their excellence and receive credit in their job. In order to provide an outline of such appropriate educational content, the major categories of educational needs and detailed specialised topics of education for the maritime community have been identified as follows:

Security-related education requirements:

- Methodology of ship security assessment. On scene survey. The threats that the vessel facing during the trip and methods to reduce the risk of this threats.

- Methods of ship security surveys and inspections. How to search the vessel or specific place for explosive devices after threat phone call.

- Instruction techniques for security training and education including security measures and procedures.

- Knowledge of current security threats and patterns.

- Recognition on a non-discriminatory basis of characteristics and behavioural patterns of persons who are likely to threaten security

- Methods of physical searches and non-intrusive inspections.

- Negotiations with terrorists.

- First Aid. General information. Care of casualty, physical examinationsymptoms and signs

Safety-related education requirements:

- Firemen Training. Familiarization with fire fighting equipments, fire suits, breathing apparatus.

- Fire Fighting. Human Risk during fire fighting. Command and control the Incident.

- Different types of fire.

- Passenger mustering and crowd management. Method of safe evacuation and mustering during emergency situation. (ex. fire). 
- Fighting Sea Pollution. Guideline Procedures to reduce the sea pollution after collision or grounding or bunkering, materials and chemicals that you can use.

General Training-related education requirements:

- Computers \& software. Training and familiarization with word, excel, outlook and power point programs

- Shipboard Management.

- Marine risk assessment. To identify the source of risk that face but also to know how to deal with them effectively. Implementing risk management strategies in the increasingly sophisticated ad competitive environment.

- Marine accident prevention and analysis. Accident prevention and Human Factor.

- Training methods

\section{Satellite-Network Architecture for Distance Learning}

We consider three distinct satellite network architectures for education applications. The rationale in supporting multiple satellite communication environments stems from: (1) the variability of expected user requirements (some users have inflexible legacy communication systems, while others can be open for very innovative deployments), and (2) the existence of a number of satellite platforms with a wide range of capabilities and cost.

The main architectures for satellite education can be identified as follows:

\subsection{VSAT Architecture}

\section{Land-to-Land VSAT Architecture}

This architecture includes a number of VSAT enabled sites and further interconnection using $\mathrm{WiFi}$ and/or WiMax wireless infrastructure for delivering the service to more users (Fig. 3). Different usage scenarios can involve point-to-point, point-tomultipoint interactive communication, live lectures and video on demand capabilities, as well as interconnection between the network sites in order to ensure the necessary collaboration between the learning groups, which is an essential eLearning element.

In the VSAT architecture, each VSAT station has its own distinct purpose. A central VSAT is needed for the broadcast of the live lectures using a fully equipped studio and the provision of the educational offline material from the eLearning platform. This VSAT station acts as a central node in the process of educational content consumption. Another VSAT antenna provides the eLearning service to users attending the course live at the tele-education hall or through a WiFi connection from other premises. The third VSAT antenna is used to facilitate the testing of VSAT and WiMax in combination with the WiFi networking. The VSAT network will have mesh topology and thus will provide the possibility of collaboration among sites.

\section{Land-to-Vessel VSAT Architecture}

It includes one or more ship(s) connected with a VSAT station at the central node with a fully equipped studio (Fig. 4). Live lectures and video on demand capabilities using IPv4 are to be integrated in the scenario. 


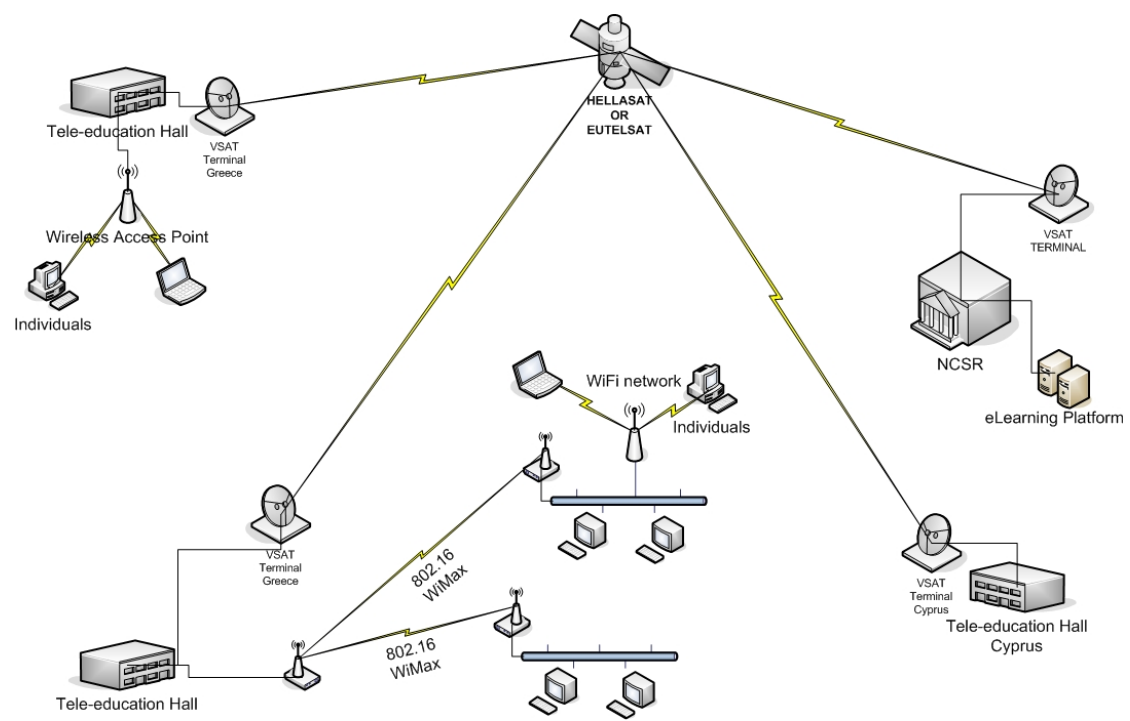

Fig. 3. VSAT networks

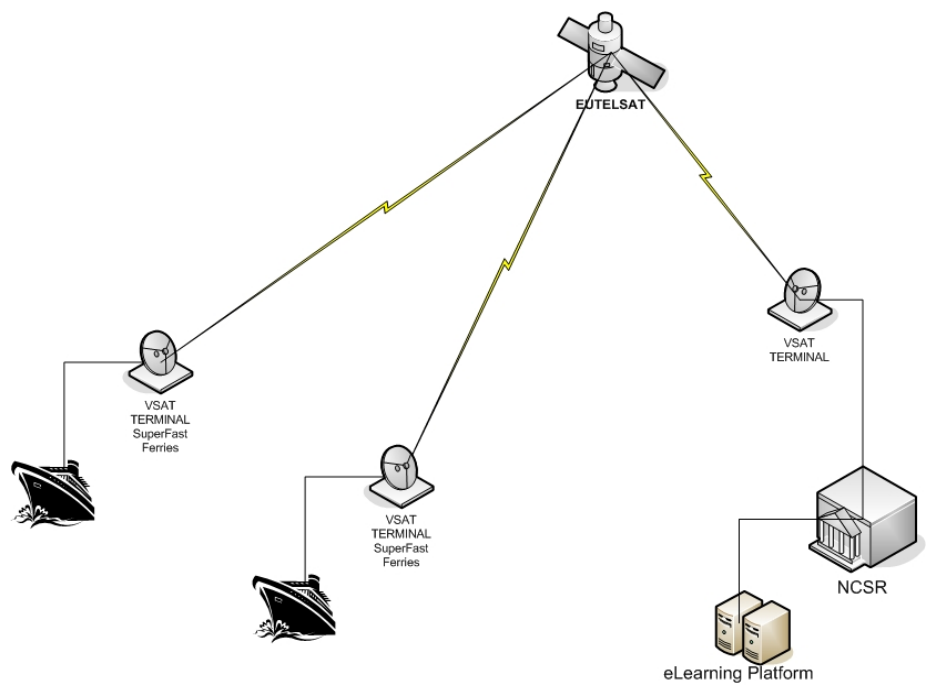

Fig. 4. Maritime network structure

\subsection{DVB-S/S2 Architecture}

This architecture involves DVB-S or DVB-S2 enabled sites for tele-training. It can support tele-training scenarios, such as point-to-point and point-to-multipoint interactive communication and collaboration, as well as live lectures and video on demand capabilities using IPv4 and IPv6 protocols (Fig. 5). 


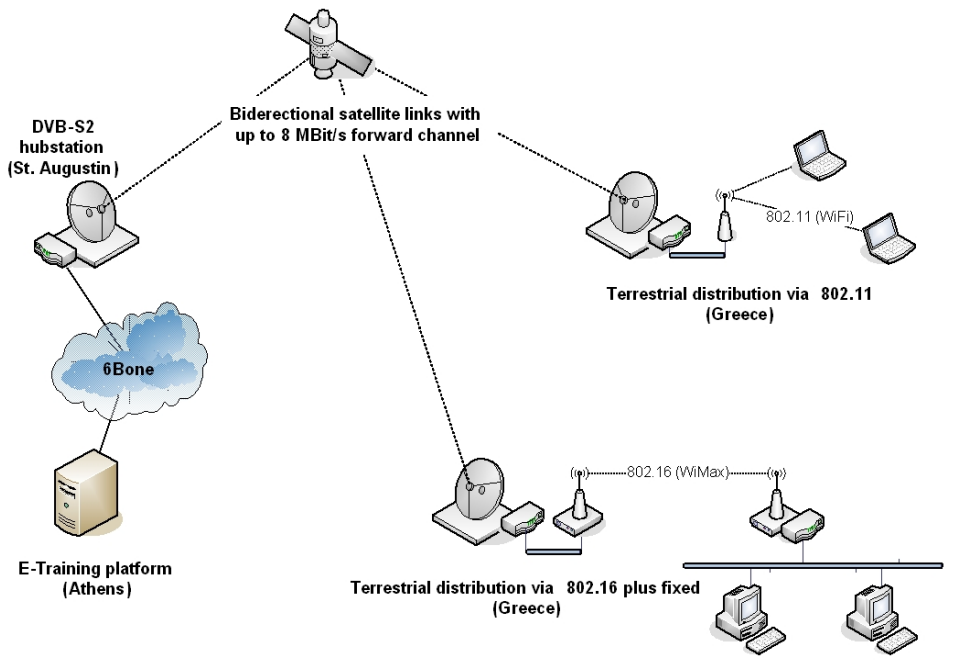

Fig. 5. DVB-S/S2 network structure

\subsection{DVB-RCS Architecture}

This architecture involves DVB-RCS enabled sites connected with the central DVBRCS platform (Fig. 6). Similarly to the previous architecture, this architecture can support live lectures and video on demand capabilities using IPv4.

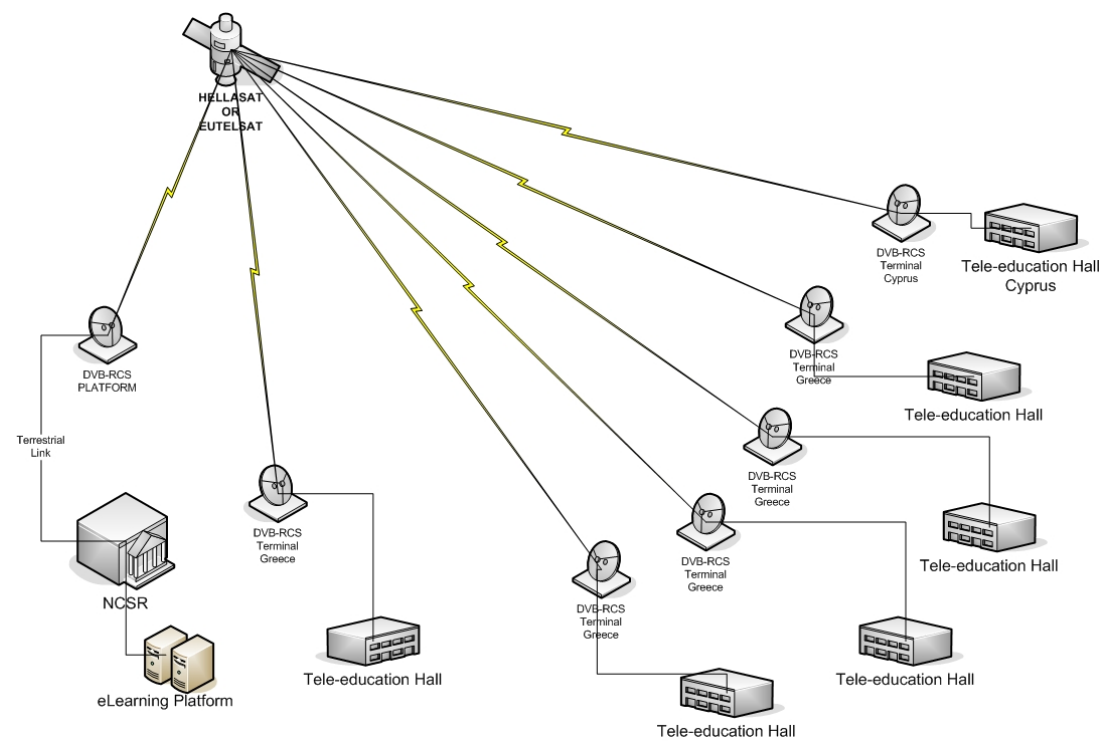

Fig. 6. DVB-RCS network structure 


\section{Distance Learning Scenarios over Satellite Networks}

Ten scenarios have been chosen to fulfill the needs of our geographically dispersed target communities. The following section lists the identified scenarios and briefly details their objectives.

\subsection{Common Room Oriented Scenarios}

Broadcast/multicast e-learning service to a common room. The primary objective of this scenario is to offer easily accessed pre-recorded educational courses targeted to audiences at remote locations, with no previous access to such services due to network connectivity restrictions. Its secondary objective is to design and present courses content based on remote audience characteristics and preferences so as to ensure immediate adoption of such services and full learners participation.

Offline feedback and $Q \& A$ session following broadcast/multicast e-learning service to a common room. The objective of this scenario is to allow smooth communication and interaction with the tutor after an e-learning session in order to resolve questions.

Virtual Classrooms with Remote Tutor; fully interactive; pre-scheduled for all participating sites or on-demand for specific sites (tutor is based on a central site). The main objective of this scenario to allow the creation, support and maintenance of a virtual classroom that provides similar tools and capabilities as in a real classroom without the physical presence of the interacting learners and teachers/tutors in the same space domain but only in the same time domain.

Webcast that is accessed from common room. The primary objective of this scenario is to promote and enable teacher-to-learner and learner-to-learner knowledge sharing.

Videoconferencing between geographically dispersed classroom sites. The core objective of this scenario is to enable learners from remote classrooms to participate live in joint lectures, seminars or workshops, interacting in more or less the same way as when physically sharing the same site.

LMS-based e-learning service that is accessed from a common tele-education room. The main objective of this scenario is to offer easily accessed educational courses targeted to audiences at remote locations, with no access to such services due to network connectivity restrictions. Its secondary objective is to design, configure, manage and present courses content and descriptions based on remote audience characteristics so as to ensure immediate adoption of such services and full audience participation

\subsection{Home Oriented Scenarios}

LMS-based e-learning service that is accessed from home. The primary objective of this scenario is to offer easily accessed educational courses targeted to audiences at remote locations, with no access to such services due to network connectivity restrictions. The secondary objective for this scenario is to design and present courses 
content based on remote audience characteristics so as to ensure immediate adoption of such services and full audience participation.

Access to Virtual classroom from home. The first objective of this scenario is to allow the creation, support and maintenance of a virtual classroom that provides similar tools and capabilities as in a real classroom without the physical presence of the interacting learners and teachers/tutors in the same space domain but only in the same time domain. The second objective is to provide the flexibility to the users to join the virtual classroom from home, and the final objective is build a Virtual Classroom environment that can facilitate standalone users to communicate whenever they want with others co-workers or users from their homes in order to be informed for their work sector or to be trained / educated.

Webcast that is accessed from home. The main objective of this scenario is to enable and promote teacher-to-learner and learner-to-learner knowledge sharing from home.

Videoconferencing from home. The objective of this scenario is to enable learners from their homes to participate live in joint lectures, seminars or workshops.

\section{Satellite-Enabled Education Services}

Starting from the user requirements and the scenarios presented in the previous sections, a number of services can be identified. The process of identification of these services is informed by the previously derived scenario specifications.

\subsection{Virtual Classroom Service}

A virtual classroom is defined as a computer accessible, on-line learning environment intended to fulfil many of the learning facilitation roles of a physical classroom. It provides a distributed learning environment at any time, any place and at any pace.

By using Virtual Classrooms, one can hold face-to-face conversations and collaborate with co-workers around the world. The features supported by these tools are concentrated on the following topics:

- Video and Audio Conferencing

- Chat

- File Transfer

- Program Sharing

- Whiteboard

- Remote Desktop Sharing

These features can be used in a point-to-point connection through the predefined networks topologies to create multi-user connections from different sites to collaborate in a conference or presentation.

The types of content that can be delivered in a virtual classroom environment can be categorized into the following functionalities: 
- Lecture Material (Course notes provided by the tutor)

- Embedded Tools (Simulations, spread sheets, calculators, etc.)

- Real time Video and Audio

- Note Taking and Annotation

- Major Exams

- Quizzes (Embedded in the coursework)

- Homework (Written material, worked problems)

- Reading Assignments (Instructor guide to readings and actual readings)

- Collaborative Projects (Teams and team projects)

- Chat and Discussion

- Messages and Email

- Feedback, Questions, and Course Evaluation

\subsection{Learning Content Management System (LCMS) Service}

Among the services that have been identified as relevant to the scope and objectives of the satellite-enabled education is the deployment of a Learning Content Management Service. An LCMS supports the authoring, publication and management of learning content, including multimedia content adaptation aspects, from the viewpoint of the system administrator and author but at the same time it will provide for personalised access to combined Learning Objects (LOs) in a Learning Path that suits best the learners of the participating communities.

An LCMS typically accommodates LOs with the following types of content:

- Video (both high quality and low quality video)

- Audio (high quality)

- Presentations (combining different formats)

- Images

- Documents

- Assignments (for both individual learners or groups of learners)

- Tests (deployed for access via Web browsers)

The above formats seem to be able to cover requirements for content to be delivered to the computers of individual learners or for content to be delivered to a classroom with many learners present over a variety of access networks and available bandwidth.

It is envisaged that the LCMS will be combined with content adaptation systems to support further personalised and quality assured access to learning solutions by the learners. Content adaptation will consist of both pre-configuring high quality content to a variety of formats, generating associated metadata descriptions to describe each content variation (pre-adaptation stage), as well as research on scalable content formats and MPEG-21 profiles generation, as well as the design of resource and metadata adaptation engines, to account for dynamic content adaptation throughout the transmission and reception path. 


\subsection{Tele-conferencing Service}

This is a core service enables live sessions connecting many geographically dispersed sites, either ad-hoc or prearranged manner. The functionality offered by a teleconferencing service is similar to that of the Virtual Classroom, however it is more focused on live interaction and more flexible with regard to time scheduling and site participation, but with limitations in terms of delivering materials. Tele-conferencing can be defined as bi-directional communication between two or more groups, or between three or more individuals, who are in dispersed sites.

Depending on the interaction capabilities offered, there are a number of teleconferencing modalities ranging from plain audio to fully multimedia-enabled. For example, small, quick and short tele-meetings can be held "over the phone" via an audio conference. The following description considers a full service, which is both audio- and video-enabled. It does not include teleconferencing intended for delivering content/LOs in an integrated environment of multiple sites or in a Virtual Classroom, as this is covered in the definition of other Services.

The remote groups (learning communities) or the individual learners from their homes get together to participate in the educational activities (e.g. attend seminars/workshops or meetings/conversations, and collaborate with colleagues/ fellow learners at other sites). Transmission of live video to and from the set-top box or PC is performed bi-directionally over a satellite network, and possibly also via wireless LAN/MAN connection. The teleconference is launched at each site by logging in to a multipoint web service at the desired time. The sessions can be either arranged "adhoc" or prescheduled.

Such a service attains the following features:

- Real time Video and Audio (multimedia networking)

- Session Scheduling tool

- Collaboration tools (Teams and team projects)

- Chat and Discussion

- File Transfer

- Program/Application Sharing

- Whiteboard

- Shared Notepad

- Remote Desktop Sharing

- Document camera

Commercial Off-the-shelf teleconferencing solutions for implementation

A number of platforms and tools can be used depending on the specific user needs:

- The Isabel conferencing tool [6] can support any possible case of delivery, providing high speed connections with many users from different geographically disparate sites.

- In the case of just one point-to-point connection with very basic functionality, a simpler tool such as Microsoft's NetMeeting [7] can also be used.

- For audio conferencing, less demanding applications based on Voice-over-IP platforms would be sufficient. 


\subsection{Webinar/Webcast Service}

This service consists of a unidirectional, live or pre recorded, transmission of content from one site to the rest of remote sites. The date and details of each session should be announced previously in order for the receiving site to tune in properly.

The main actors that interact in the educational process could be teachers and learners, or just learners among them. There are no restrictions on the communities that could be able to use this service; In principle any community (including agrarian and maritime) can benefit from it.

While the content can be pre recorded at the transmitting site, this service does not involve on-demand download. In that way the receiving site can not distinguish if the content being played is live or pre recorded. The only difference in this case is at the transmitting site.

The content could be received at a common room or at the learner's home. Both scenarios are pretty similar. The main difference is the need for a more sophisticated common room setup. It is expected to use common-of-the-shelves player software at the receiving site.

\subsection{Other Services}

Another potential service that accommodates the needs of already defined e-learning scenarios is the "Broadcast/multicast e-learning service". In such a service, prerecorded educational content is either broadcasted over satellite core and access networks (DVB-S2, VSAT) or multicasted over broadband satellite and wireless IPbased access networks (DVB-RCS, Wi-Fi, Wi-Max) respectively to remote groups of learners gathered in a tele-education hall, equipped with receiving satellite antennas and hubs or wireless access points, set-top boxes or PCs, video projectors, VCRs, TV sets, microphones and speakers. The session is initiated either at pre-scheduled times or by the course coordinator and involves initially passive viewing of the educational course and later on communication and interaction possibilities for Q\&A sessions. Such a service involves use of an LMS/LCMS to store educational pre-recorded content, configured in a variety of formats to accommodate differentiated networking bandwidths. Associated annotations/descriptions along with course information and scheduling are stored along with the content into the LMS/LCMS. Access to this service is feasible either through a purely satellite network and client terminals (VSAT, DVB-RCS), or through a core satellite network interlinked with broadband wireless access networks (DVB-S2/VSAT + WiFi/WiMax). Broadcasting or multicasting is implemented based on IP protocols for streaming over Internet such as IPv4 and IPv6. Return channel communication for the Q\&A session takes advantage of the bidirectional capabilities of DVB-RCS and VSAT satellite networks, while in the case of unidirectional DVB-S2 satellite networks, the possibility to use SCPC for backchannel communication is considered. User means of communication and interaction with the tutors/coordinator are through emails, chats, forums, audio conferences, etc.

Several other types of services may be applicable in case that targeted community requirements are not met by the currently defined ones, or system and infrastructural requirements are faced with technology limitations and feasibility risks. 


\section{Conclusions}

This paper has discussed the need for satellite-based tele-education in the context of providing tele-training services to professional communities, which are distributed in remote areas. The satellite-based service requirements have been identified for two important communities (agricultural and maritime). Three network architectures have been described that fulfill the system requirements. A number of usage scenarios have been identified and critically examined in terms of their applicability and potential benefits to the two communities. Finally, a raft of services has been proposed to implement the envisaged scenarios based on designed satellite architectures. It is the authors' strong belief that the development and wider adoption of such satellite-based services will extend the benefits of ICT-enabled education to an even larger proportion of the workforce worldwide. It is also expected that that emergence of such learner-oriented systems for distance education via satellite communication will lead to sustainable solutions to the problem of delivering education to geographically dispersed learner communities in the developing countries, most of which suffer from a lack of any network infrastructure.

\section{Acknowledgments}

The authors are grateful to Mathias Kretschmer, Andreas Voigt, Gabriel Huecas Fernandez-Toribio, Carlos Barcenilla, Vouyioukas Demosthenes and Dimitra Pappa for their valuable comments and for fruitful discussion. The presented work is supported by the Aeronautics and Space programme of European Commission, as part of the BASE2 project (Contract no.: 516159)

\section{References}

[1] Urdan. T., Weggen, C.: Corporate E-Learning: Exploring a New Frontier: http:// www. spectrainteractive.com/pdfs/CorporateELearingHamrecht.pdf (Accessed on 3 April 2006)

[2] Hambrech, W.R.: Corporate e-Learning: "Exploring a new Frontier" http://www. learnchamp.com/upload/e-Learning _Exploring_New_Frontier.pdf (Accessed on 3 April 2006)

[3] Koskinen, T., Stergioulas, L.K., and Denoual, Y.: European Roadmap for Professional eTraining, Barcelona (2004).

[4] Stergioulas, L.K., Kamtsiou, V. and Koskinen, T.: A roadmapping framework for technology-enhanced professional training In: Proceedings of the 8th IFIP World Conference on Computers in Education (WCCE '05), Cape Town, South Africa, 4-7 July 2005.

[5] Santos, M.; Tendencias en la formación con medios digitales: el e-Learning. http://www.unav.es/digilab/ric/textos/e_learning.html (Accessed 3 April 2006)

[6] Isabel: Isabel Technical Documentation. Available on http://www.agora-2000.com/ products/isabel/documentation.html (Accessed on 12 April 2006)

[7] Netmeeting: Microsoft Netmeeting Features, Available at http://www.microsoft.com/ windows/NetMeeting/Features/default.ASP (Accessed on 12 April 2006) 